\title{
Centrality without Indices: Partial Rankings and Rank Probabilities in Networks
}

DOI:

10.1016/j.socnet.2017.12.003

\section{Document Version}

Accepted author manuscript

Link to publication record in Manchester Research Explorer

\section{Citation for published version (APA):}

Schoch, D. (2018). Centrality without Indices: Partial Rankings and Rank Probabilities in Networks. Social Networks, 54, 50-60. https://doi.org/10.1016/j.socnet.2017.12.003

\section{Published in:}

Social Networks

\section{Citing this paper}

Please note that where the full-text provided on Manchester Research Explorer is the Author Accepted Manuscript or Proof version this may differ from the final Published version. If citing, it is advised that you check and use the publisher's definitive version.

\section{General rights}

Copyright and moral rights for the publications made accessible in the Research Explorer are retained by the authors and/or other copyright owners and it is a condition of accessing publications that users recognise and abide by the legal requirements associated with these rights.

\section{Takedown policy}

If you believe that this document breaches copyright please refer to the University of Manchester's Takedown Procedures [http://man.ac.uk/04Y6Bo] or contact uml.scholarlycommunications@manchester.ac.uk providing relevant details, so we can investigate your claim.

\section{OPEN ACCESS}




\title{
Centrality without Indices: Partial Rankings and Rank Probabilities in Networks ${ }^{\text {th }}$
}

\author{
David Schoch ${ }^{\mathrm{a}, *}$ \\ ${ }^{a}$ Chair of Social Networks, ETH Zurich, Switzerland
}

\begin{abstract}
We present an alternative approach to assess centrality in networks which does not rely on traditional indices. The work is based on neighborhood-inclusion, a partial ranking inducing relation of nodes, which was shown to be preserved by many existing centrality indices. As such, it can serve as the shared basis for centrality in networks. We argue that evaluating this partial ranking by itself allows for a generic assessment of centrality, avoiding several pitfalls that can arise when indices are applied. Additionally, we illustrate how to derive further partial rankings and introduce some probabilistic methods to, among others, compute expected centrality ranks of nodes.
\end{abstract}

Keywords: Network centrality, Neighborhood-inclusion, Centrality indices, Rank probabilities, Partial Orders

\section{Introduction}

Network centrality is commonly defined in terms of indices, assigning numerical scores to the nodes of a network. These scores induce a ranking which is meant to reflect the structural importance of entities comprising a network. There is, however, little agreement on what constitutes "structural importance" and many indices exist, assessing it on various levels (Lü et al., 2016). Much effort was put into conceptual clarifications (Freeman, 1979), axiomatizations (Sabidussi, 1966; Nieminen, 1974; Ruhnau, 2000, Landherr et al., 2010; Kitti, 2012; Boldi and Vigna, 2014) and classifications (Borgatti, 2005, Borgatti and Everett, 2006), yet formal guidelines to restrict the set of possible indices or alternative methods are scarce.

Recently, Schoch and Brandes (2016) introduced a simple characterization of centrality: if an actor has the same (and possibly more) ties than another actor, it can never be less central. The authors showed that there exists a

\footnotetext{
${ }^{2}$ We gratefully acknowledge financial support from Deutsche Forschungsgemeinschaft (DFG) under grant Br 2158/6-1

* Corresponding author

Email address: david.schoch@gess.ethz.ch (David Schoch)
} 
partial ranking of nodes based on neighborhood-inclusion which is preserved by many known centrality indices. This finding can be used to characterize possible centrality rankings (hence indices) as the set of rankings extending the partial ranking given by neighborhood-inclusion.

Preorders as given above are a simple and well studied mathematical structure (Davey and Priestley, 2002, Grätzer, 2002), emerging in many different fields where objects need to be put in order. A prime example provides the field of multi-criteria decision making (MCDM) (Belton and Stewart, 2002, Triantaphyllou, 2013). Given a set of alternatives valued by a set of criteria, the objective is to find the "best" alternative. This is commonly done using indices aggregating the criteria of each alternative into a preference ranking. The preference rankings are supposed to preserve an intrinsic dominance order, that is if an object is better for each criterion than another, it should always be preferred to the dominated one. In a sense, the idea of these indices is conceptually related to centrality indices.

While indices are well established to build preference rankings, they are perpetually under scrutiny for various fundamental issues. Stewart (1992) summarizes that there exists "a plethora of approaches", where "some of these are ad hoc, and largely unjustified on theoretical and/or empirical grounds.". Patil and Taillie (2004) argue that indices are typically "adopted on grounds of mathematical convenience or simplicity" without proper justifications. Triantaphyllou and Mann (1989) uncovered the infamous decision making paradox which states that different index based decision methods can yield different results when fed with the same data. Put in other terms, the choice of index affects the choice of the most preferred alternative. The paradox is also related to the problem of rank reversals, i.e. the most preferred alternative changes when an actually inferior alternative is added. This issue was first described by Belton and Gear (1983).

The outlined issues in MCDM are fundamentally linked to network centrality. We too are faced with a plethora of indices that are, according to Freeman (1979), "often unnecessarily complicated", "absolutely unintelligible from any theoretical perspective whatever", or "tend to add unnecessary and confusing complications that make them difficult to interpret." Moreover, choosing an appropriate index in an empirical setting becomes a daunting task when faced with the overabundance of indices. This may foster trial and error approaches, probing different indices until a satisfactory result is obtained.

Many alternative methods exist to analyse and determine (partial) rankings which do not necessarily rely on indices. In this work, we focus on methods that build upon an intrinsic ordering of some pairs of objects. These methods range from simple analyses of their structure (Patil and Taillie, 2004, Pavan and Todeschini, 2004), how to handle incomparability (Brüggemann and Carlsen, 2014: Bartel and Mucha, 2014), constructing non-numerical rankings (Fishburn and Gehrlein, 1975, Janicki, 2008, 2009) to computing all possible rankings that preserve the intrinsic ordering (Bubley and Dyer, 1999, Habib et al., 2001). The latter are mostly of interest to derive (relative) rank probabilities and expected ranks (Brüggemann et al., 2003, 2004, 2005, De Loof et al., 2006, 2008, De Loof, 
2009).

In the upcoming parts, we illustrate how these existing methods can be used in the context of network centrality, offering an alternative or complementary approach to centrality indices. We start in Section 2 by introducing the intrinsic ordering of nodes based on neighborhood-inclusion. We illustrate how to decompose indices into three building blocks which facilitate further theoretical considerations. Lastly, we show how the neighborhood-inclusion preorder can be extended to denser partial rankings. The remainder of the work builds on the theoretical results from this section. In Section 3 , we offer first alternatives to indices by introducing Hasse diagrams and assessing rank ambiguities with rank intervals. Subsequently, we introduce probabilistic methods for centrality in Section 4. These include relative rank probabilities (How likely is it, that a node is more central than another?) and expected ranks of nodes (How central is a node expected to be considering all possible rankings?). In Section 5, we illustrate how the presented methods can be employed in empirical research. Finally, we end with some concluding remarks and a discussion in Section 6 .

\section{Centrality indices and partial rankings}

Centrality is commonly defined in terms of mappings $c: V \rightarrow \mathbb{R}_{\geq 0}$ assigning real valued scores to the set of nodes $V$ of a graph $G=(V, E)$ and are interpreted as

$$
c(u)>c(v) \Longleftrightarrow u \text { is more central than } v .
$$

The most widely used indices are degree, betweenness (Freeman, 1977) which was earlier introduced as rush by Anthonisse (1971), closeness (Bavelas, 1948. Sabidussi, 1966), and eigenvector centrality which was introduced for the first time by Wei (1952), later generalized by Berge (1958) and reintroduced by Bonacich (1972). Recently, Schoch and Brandes (2016) showed that there exists a shared basis among a large group of indices, including these prototypical ones, by a comparison of neighborhoods in graphs. The idea is based on a decomposition of indices into the following three generic steps:

(1) Deriving indirect relations via path algebras.

(2) Defining vertex positions via coordinates evaluating indirect relations.

(3) Defining centrality scores by aggregating values of positions.

In this section, we formally introduce this framework and illustrate how each of these steps leads to the definition of a partial ranking on networks. Additionally, we illustrate how this framework can be used to derive additional partial rankings, which are preserved by specific classes of indices. 


\subsection{Indirect relations}

Indirect relations on graphs can be characterized with so called path algebras based on the algebraic structure of semiring $\AA^{1}$

Definition 1. An algebraic structure $(S, \oplus, \odot, \overline{0}, \overline{1})$ on a set of values $S$ is called $a$ semiring if and only if

(i) $\oplus, \odot: S \times S \rightarrow S$ are closed and associative

(ii) $\overline{0}, \overline{1} \in S$ are neutral elements of $\oplus$ and $\odot$, respectively

(iii) $\overline{0}$ is absorbing for $\odot$

(iv) $\oplus$ is commutative and $\odot$ distributes over $\oplus$

Path algebras characterize indirect relations on graphs $G=(V, E)$ for vertices $s, t \in V$ by associating a value from a semiring with every $(s, t)$-path and then aggregating over all such paths. We associate each edge with an element $\bar{e} \in S$, the edge value, so that we can characterize a graph $G=(V, E)$ by a matrix $A \in S^{V \times V}$ with entries

$$
a_{s t}= \begin{cases}\bar{e} & \{s, t\} \in E \\ \overline{0} & \text { otherwise }\end{cases}
$$

for all $s, t \in V$. An $(s, t)$-path $P$ with vertex sequence $s=v_{0}, v_{1}, \ldots, v_{k-1}, v_{k}=t$ is then evaluated by

$$
a(P)=\bigodot_{i=1}^{k} a_{v_{i-1}, v_{i}},
$$

where $a(P)=\overline{1}$ if $k=0$. The indirect relation between $s$ and $t$ is then obtained from

$$
a_{s t}^{*}=\bigoplus_{i=1}^{\infty} a(P),
$$

where all possible $(s, t)$-paths are aggregated. If no such path exists, $a_{s t}^{*}=\overline{0}$ holds. The term $a_{s t}^{*}$ is also known as the closure of $a_{s t}$. A convenient joint formulation can be derived in terms of matrices. Let $A \in S^{V \times V}$ be a matrix as defined above, and let $1 \in S^{V \times V}$ be the matrix with entries $\overline{1}$ on the diagonal and $\overline{0}$ elsewhere. This formulation gives rise to a semiring of matrices $\left(S^{V \times V}, \oplus, \odot, \mathbf{0}, \mathbf{1}\right)$, where $\oplus$ and $\odot$ replace the usual matrix addition and multiplication. By defining $A^{0}=\mathbf{1}$ and $A^{k}=A \odot A^{k-1}$ for $k \geq 1$, we obtain the closure $A^{*}=\bigoplus_{k=0}^{\infty} A^{k}$ with entries $a_{s t}^{*}$ as above.

For later purposes, we need two additional properties of semirings. The first defines an order relation on the set $S$.

${ }^{1} \mathrm{~A}$ comprehensive introduction of semirings and path algebras is given by Gondran and Minoux (2008). 
Definition 2. The canonical preorder of a semiring $(S, \oplus, \odot, \overline{0}, \overline{1})$ is given by

$$
a \leq b \text { if } a \oplus c=b \text { for some } c \in S \text {. }
$$

The second property captures the intuition that moving away from an actor by an additional indirect relation can only reduce the value of a relationship.

Definition 3. Let $(S, \oplus, \odot, \overline{0}, \overline{1})$ be a semiring and $\bar{e} \in S$ be an edge value. The semiring is called decreasing if

$$
\bar{e} \odot a \leq a
$$

holds for all $a \in S$.

As an example, consider the geodesic semiring $\left(\mathbb{N}_{0} \cup \infty\right.$, min, $\left.+, \infty, 0\right)$ with edge value $\bar{e}=1$. By concatenation with Equation (1) and aggregation via Equation (2), we obtain entries $a_{s t}^{*}=\operatorname{dist}(s, t)$ and thus the distance matrix as the closure $A^{*}$ of the corresponding matrix semiring. The geodesic semiring is decreasing, since adding an edge to a path increases the distance. It should be noted though that the preorder on the semiring is the reverse order of natural numbers. That is, for $a, b \in \mathbb{N}_{0}$ with $a \geq b$, we have $a \leq b$ in $S$.

Another important semiring for indirect relations, the geodetic semiring, was introduced by Batagelj (1994). We set $S=\left(\mathbb{N}_{0} \cup \infty\right) \times \mathbb{N}_{0}, \overline{0}=(0, \infty), \overline{1}=(0,1)$ and for $(a, b),(c, d) \in S$ we define

$$
\begin{aligned}
& (a, b) \odot(c, d)=(a+c, b \cdot d) \\
& (a, b) \oplus(c, d)=\left(\min \{a, c\},\left\{\begin{array}{ll}
b & a<c \\
b+d & a=c \\
d & a>c
\end{array}\right) .\right.
\end{aligned}
$$

By assigning the edge value $\bar{e}=(1,1)$, we obtain $a_{s t}^{*}=(\operatorname{dist}(s, t), \sigma(s, t))$, where $\sigma(s, t)$ is the number of shortest $(s, t)$-paths. The canonical preorder on the geodetic semiring translates to

$$
(a, b) \leq(c, d) \Longrightarrow(a>c) \vee(a=c \wedge b \leq d) .
$$

The semiring is also decreasing since

$$
(1,1) \odot(a, b)=(a+1, b) \leq(a, b) .
$$

Walk-based centrality indices, such as eigenvector centrality, Katz status (Katz, 1953), subgraph centrality (Estrada and Rodríguez-Velázquez, 2005b) which is given by

$$
c_{s c}(u)=\sum_{k=0}^{\infty} \frac{A(G)_{u u}^{k}}{k !}
$$

where $A(G)^{k}$ is the $k$-th power of the adjacency matrix of a graph $G=(V, E)$, and total communicability (Benzi and Klymko, 2013), defined as

$$
c_{t c}(u)=\sum_{t \in V} \sum_{k=0}^{\infty} \frac{A(G)_{u t}^{k}}{k !},
$$


can also be expressed via a joint semiring formulation. Denote by $\omega_{s t, k}$ the number of $(s, t)$-walks with $k$ edges, where $s, t \in V$ and $k \in \mathbb{N}_{0}$. This sequence of walk counts can be represented by the generating function $\Omega(\beta)=\sum_{k=0}^{\infty} \omega_{k} \cdot \beta^{k}$, which converges to a limit function for sufficiently small $\beta$, depending on the growth of the sequence $\left(\omega_{k}\right)_{k \in \mathbb{N}_{0}}$. Addition and convolution of sequences then define a semiring for $\beta \geq 0$ together with $\overline{1}=(1,0,0, \ldots), \overline{0}=(0,0, \ldots)$, and edge value $(0,1,0,0, \ldots)$. The resulting indirect relations are given by $a_{s t}^{*}=$ $\left(\omega_{s t, 0}, \omega_{s t, 1}, \ldots\right)$. Subgraph centrality and total communicability are based on exponential generating functions $\omega(\beta)=\sum_{k=0}^{\infty} \frac{\omega_{k}}{k !} \cdot \beta^{k}$, which form a semiring with the same neutral elements and edge value as $\Omega(\beta)$. Both semirings are not decreasing, but can be reformulated based on prefix sums $\omega_{s t}^{(k)}=\sum_{i=0}^{k} \omega_{s t, i}$ to form decreasing semirings. More details are provided in Schoch and Brandes (2016).

\subsection{Positions and indices}

The indirect relations $A^{*}$ derived from path algebras form the basis to build various centrality indices. The geodesic semiring, for instance, directly provides the relations needed for closeness centrality. Occasionally, however, further specifications are necessary since indirect relations have to be transformed into positional vectors of real numbers. For this purpose, we need to define a transformation function $\tau$, which maps values from the closure of a semiring to the set of non-negative real numbers. In the case of closeness, $\tau$ is simply the identity function and the positional vector of all vertices $u \in V$ is given by

$$
\operatorname{pos}(u)=(\operatorname{dist}(u, t))_{t \in V}
$$

The closure of the geodetic semiring can be transformed into dyadic dependencies

$$
\delta(s, u)=\sum_{t \in V} \begin{cases}\frac{\sigma(s, u) \cdot \sigma(u, t)}{\sigma(s, t)} & \operatorname{dist}(s, t)=\operatorname{dist}(s, u)+\operatorname{dist}(u, t) \\ 0 & \text { otherwise, }\end{cases}
$$

for all $s, u \in V$ and we obtain the positional vector

$$
\operatorname{pos}(u)=(\delta(s, u))_{s \in V}
$$

The final step in building indices is to summarize these vectors into a score. The most convenient choice is summing up all entries. Betweenness, for instance, is defined as the sum of dyadic dependencies, $c_{b}(u)=\sum_{s \in V} \delta(s, u)$. However, other summarizations are feasible as well. Subgraph centrality is obtained via projection to entry $\omega_{u u}(\beta)$ in the semiring of exponential generating functions based on prefix sums. The aggregation step can also include a rank reversing function, such as for closeness where the inverse of the sum is taken, or for eccentricity (Hage and Harary, 1995) which is defined as the inverse of the maximum entry of the positional vector given by distances. These rank reversing steps usually occur for "costly" relations. 
The presented steps establish a general framework for centrality indices, given that they can be derived from a decreasing path algebra and a monotonic transformation step. The main focus of the framework is set on deriving indirect relations that are supposed to be indicative for centrality. In the following, we illustrate how the third and also the second step can be omitted by defining partial rankings on the set of nodes. This allows to put more emphasis on the choice of appropriate indirect relations than on the choice of transformations and indices. Note that when speaking of centrality indices from now on, we exclusively refer to those that meet the requirements of the presented framework.

\subsection{Neighborhood-inclusion preorder}

The neighborhood $N(u)$ of a node $v \in V$ in a simple undirected graph $G=(V, E)$ is defined as $N(u)=\{w:\{u, w\} \in E\}$. Further, the closed neighborhood $N[u]$ is given by the union of $N(u)$ and $\{u\}$. The following definition introduces a preorder on the set of vertices based on neighborhoods.

Definition 4. Let $G=(V, E)$ be a simple undirected graph and $u, v \in V$. The relation

$$
u \leq v \text { if } N(u) \subseteq N[v]
$$

indicates that the neighborhood of $v$ includes that of $u$.

If $u \leq v$, we say that $u$ is dominated by $v$. If neither $u \leq v$ nor $v \leq u$ holds, we say that $u$ and $v$ are incomparable. Throughout this work we refer to this dominance relation as neighborhood-inclusion preorder or partial ranking induced by neighborhood-inclusion. The following theorem is due to Schoch and Brandes (2016) and relates this preorder to a class of centrality indices.

Theorem 1. Let $G=(V, E)$ be a simple undirected graph, and $c: V \rightarrow \mathbb{R}_{\geq 0}$ a centrality index that is obtained from a decreasing path algebra via monotone quantification and summarization of positions. Then, for all $u, v \in V$,

$$
u \leq v \Longrightarrow c(u) \leq c(v)
$$

The intriguing part of the theorem is that although neighborhood-inclusion is a very localized property, it is preserved by many indices which take the whole path or walk structure of a graph into account. While it certainly does not cover all centrality indices, it at least includes those most commonly used and any (not necessarily known) index which can be reduced to the presented framework. That is, indices that can be derived from the closure $A^{*}$ of a decreasing and preordered semiring paired with some monotonic transformation function $\tau$ and a summarization method such as summation, maximum or projection. All indices mentioned thus far fall into this category.

Figure 1 shows an example graph and the neighborhood-inclusion preorder as a directed graph. This graph is used as a running example throughout this paper. The centrality scores for the indices degree, betweenness, closeness, and eigenvector centrality are given in Table 1 . 


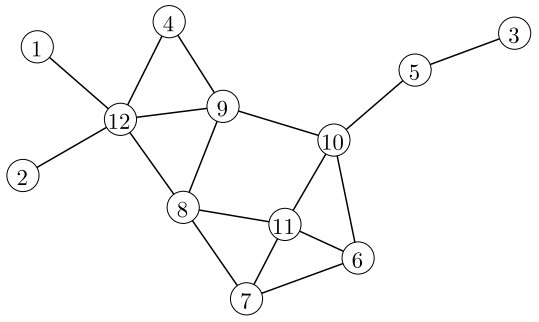

(a) Graph
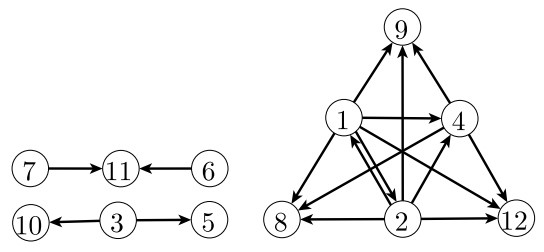

(b) Dominance relations

Figure 1: Example graph and the neighborhood-inclusion preorder as a directed graph. An arc $(u, v)$ is present, if $N(u) \subseteq N[v]$ holds.

Table 1: Centrality scores for the graph shown in Figure 1(a).

\begin{tabular}{rrrrr}
\hline Node & Degree & Betweenness & Closeness & Eigenvector \\
\hline 1 & 1 & 0.00 & 0.032 & 0.24 \\
2 & 1 & 0.00 & 0.032 & 0.24 \\
3 & 1 & 0.00 & 0.026 & 0.07 \\
4 & 2 & 0.00 & 0.038 & 0.50 \\
5 & 2 & 10.00 & 0.036 & 0.26 \\
6 & 3 & 1.50 & 0.038 & 0.74 \\
7 & 3 & 1.50 & 0.040 & 0.78 \\
8 & 4 & 11.17 & 0.048 & $\mathbf{1 . 0 0}$ \\
9 & 4 & 17.17 & $\mathbf{0 . 0 5 3}$ & 0.91 \\
10 & 4 & $\mathbf{2 1 . 8 3}$ & 0.050 & 0.82 \\
11 & 4 & 4.50 & 0.045 & 0.96 \\
12 & $\mathbf{5}$ & 20.33 & 0.048 & 0.83 \\
\hline
\end{tabular}

Observe that the dominance relations shown in Figure 1(b) are preserved for each index. For instance, the scores of node 11 are always greater than the scores of node 6 and 7 . If pairs of nodes are incomparable, then their order depends on the chosen index. Node 12 is more central than 11 according to degree and closeness, but less central for betweenness and eigenvector centrality. The dominance relations thus form an intrinsic ordering of nodes, irrevocable by centrality indices that fulfill the requirements of Theorem 1. Indices of such kind thus merely extend this partial ranking into one possible ranking. Note that we deliberately discard the actual scores of indices and are only concerned with their induced ranking, that is the rank order of nodes according to the scores. While this reduces centrality to an ordinal scale, it allows for a more general treatment. Different indices may induce the same ranking ${ }^{2}$ and for each possible ranking, we can find an index that induces said ranking. As we

\footnotetext{
${ }^{2}$ In fact there are infinitely many that do so by transforming the index monotonically.
} 
illustrate later, the number of possible rankings can be enormous. As a rough indicator, the more incomparable pairs exist, the more freedom indices have to rank nodes differently (Schoch et al., 2017).

In the following, we show how to extend the neighborhood-inclusion preorder in order to reduce the number of possible centrality rankings in applications when the indirect relation of interest is clear, using the proposed framework.

\subsection{Extended dominance concepts}

The last subsection showed that neighborhood-inclusion is preserved for indices based on different graph-theoretic concepts, such as walk counts or geodesic distances. This generality, however, comes at the expense of specificity in empirical settings. Following the proposed framework, the first step in a centrality related analysis is to derive indirect relations which are indicative for centrality. Once derived, an optional transformation function can be employed for further specifications. Since the only requirement to be met is monotonicity, there remains a wide array of possibilities. Thus, even if the indirect relation is evident, it is still possible to define many different indices based on said relations. As an example, consider the indices given in Table 2, showing a selection based on monotonic transformations of geodesic distances.

Table 2: Distance based centrality indices. From top to bottom: classic closeness, harmonic closeness, integration, residual closeness, decay centrality and generalized closeness.

\begin{tabular}{|c|c|c|}
\hline Index & Transformation & Reference \\
\hline$\frac{1}{\sum_{t \in V} \operatorname{dist}(i, t)}$ & $\tau(x)=\mathrm{id}(x)$ & Sabidussi (1966) \\
\hline$\sum_{t \in V} \frac{1}{\operatorname{dist}(i, t)}$ & $\tau(x)=\frac{1}{x}$ & Harris $(1954)$ \\
\hline$\sum_{t \in V} 1-\frac{\operatorname{dist}(i, t)-1}{\operatorname{diameter}(G)}$ & $\tau(x)=1-\frac{x-1}{C}$ & Valente and Foreman 1998 \\
\hline$\sum_{t \in V} 2^{-\operatorname{dist}(i, t)}$ & $\tau(x)=2^{-x}$ & Dangalchev (2006) \\
\hline$\sum_{t \in V} \alpha^{\operatorname{dist}(i, t)} \quad \alpha \in(0,1)$ & $\tau(x)=\alpha^{x}$ & Jackson $(2010)$ \\
\hline$\sum_{t \in V} \operatorname{dist}(i, t)^{-\alpha} \quad \alpha \geq 0$ & $\tau(x)=x^{-\alpha}$ & Agneessens et al. (2017) \\
\hline
\end{tabular}

The reasons for the existence of various indices based on the same indirect relation are manifold. They include purely technical reasons, for instance applicability on disconnected networks, but also empirical ones since using different transformations may lead to a better fit for observed phenomena.

\footnotetext{
${ }^{3}$ The authors also consider negative values for $\alpha$ which we here exclude, since they are not in accordance with the neighborhood-inclusion preorder and the intuition that lower distances are preferable.
} 
To avoid a data fitting approach and probing different transformations, we here suggest to consider an extended version of neighborhood-inclusion which is preserved by any monotonic transformation. As a first step, we decompose the implication given in Theorem 1 into

$$
u \leq v \stackrel{(i)}{\Longrightarrow} a_{u t}^{*} \leq a_{v t}^{*} \quad \forall t \in V \stackrel{(i i)}{\Longrightarrow} \tau\left(a_{u t}^{*}\right) \leq \tau\left(a_{v t}^{*}\right) \quad \forall t \in V \stackrel{(i i i)}{\Longrightarrow} c(u) \leq c(v) .
$$

The terms $a_{u t}^{*}$ and $a_{v t}^{*}$ refer to the respective entries of the closure $A^{*}$ of a decreasing path algebra, $\tau$ to a monotonic transformation function and $c: V \rightarrow$ $\mathbb{R}_{>0}$ to the respective centrality index. Implication $(i)$ follows from Lemma 3 in Schoch and Brandes (2016 (ii) from the assumed monotonicity of $\tau$ and (iii) from the fact that if each entry of a vector is smaller than the same entry of another then so is the sum (maximum entry, projection to one entry) of the vector. Each step defines a new preorder on the set of vertices which increases (or at least not decreases) the number of comparable pairs of the neighborhoodinclusion preorder.

We can extend these preorders further by noticing that the summarisation step is commutativity. Thus, it does away with identities of vertices since we can rearrange the positional vectors without altering the final scores. More substantially, this leads to the assumption of homogeneity of actors in a network. A dominance relation based on these considerations is given in the following definition.

Definition 5. Let $G=(V, E)$ be a simple undirected and unweighted graph and $u, v \in V$. Further let $A^{*}$ be the closure of a decreasing path algebra on $G$. If there exists a permutation $\pi: V \rightarrow V$ such that $a_{u t}^{*} \leq a_{v \pi(t)}^{*}$ holds for all $t \in V$, then $u$ is dominated under the total homogeneity assumption on $A^{*}$, denoted by $u \leq A^{*} v$.

The relations of dominance under total homogeneity are preserved for any index $c: V \rightarrow \mathbb{R}_{\geq 0}$ based on $A^{*}$, if the transformation step involves a monotonic function. The dominance relations are conveniently checked by sorting each row of $A^{*}$ non-increasingly (or non-decreasingly for cost relations such as distances) and comparing values front to back. It is clear that

$$
u \leq v \Longrightarrow u \leq_{A^{*}} v
$$

holds but not vice versa. As such, dominance under total homogeneity constitutes a proper extension of neighborhood-inclusion for cases where the indirect relation of interest is clear. Note that both neighborhood-inclusion and dominance under total homogeneity are specific instantiations of positional dominance as introduced by Brandes (2016).

Going back to our example graph, we find that while $76 \%$ of all pairs are incomparable with neighborhood-inclusion, only $9 \%$ are incomparable with dominance under total homogeneity on the geodesic semiring. Distance based indices

\footnotetext{
${ }^{4}$ The entries $a_{u v}^{*}, a_{u u}^{*}$ and $a_{v v}^{*}$ technically need a special treatment. For ease of exposition, we omnit this here and refer to Schoch and Brandes (2016) for further details.
} 
thus have little degree of freedom to rank nodes differently, resulting in an overall high rank correlations. Table 3 illustrates that no correlation among indices from Table 2 is below 0.90 .

Table 3: Rank correlation (Kendall's $\tau$ ) of distance based indices on the example graph.

\begin{tabular}{lllllll}
\hline & $(1)$ & $(2)$ & $(3)$ & $(4)$ & $(5)$ & $(6)$ \\
\hline (1) classic & 1.00 & & & & & \\
(2) harmonic & 0.92 & 1.00 & & & & \\
(3) integration & 1.00 & 0.92 & 1.00 & & & \\
(4) residual & 0.95 & 0.97 & 0.95 & 1.00 & & \\
(5) decay $(\alpha=0.75)$ & 0.98 & 0.94 & 0.98 & 0.97 & 1.00 & \\
(6) generalized $(\alpha=1.5)$ & 0.92 & 1.00 & 0.92 & 0.97 & 0.94 & 1.00 \\
\hline
\end{tabular}

\section{Partial centrality rankings}

So far, we have introduced two partial rankings on the set of nodes of a graph. The very generic neighborhood-inclusion preorder which is at least preserved by centrality indices that fulfill the properties of Theorem 1, and the more specific dominance under total homogeneity, preserved by indices based on monotonic transformations of given indirect relations. In the following, we present several existing methods which can be used to analyze these under the premise of centrality, without necessarily resorting to indices. In this section, we present two methods to evaluate structural features of partial rankings which may be employed before any ranking is constructed.

\subsection{Hasse Diagrams}

Partial rankings can be visualized with a Hasse diagram, an alternative to the graph given in Figure 1(b). A Hasse diagram displays the transitive reduction of the dominance relation as a vertical drawing. Hence, two nodes $u$ and $w$ are connected with an upward edge if $u \leq w$ and no node $v$ exists so that $u \leq v \leq w$. Apart from this convention, there are not many strict guidelines for drawing the diagram. In the following, we introduce two drawings that can prove helpful in our context. For an extensive discussion of Hasse diagrams, see e.g. Neggers and $\operatorname{Kim}(1998)$.

The first option is to draw the Hasse in levels from top to bottom. The top level consists of nodes that are not dominated by any other node, that is $u$ is in the top level if $u \leq z v$ for all $v \in V \backslash\{u\}$. Nodes in the top level are the maximal elements of the preorder. The second level consists of the elements that become maximal if the top level is removed. This procedure is repeated until all nodes have been assigned to a level. The second option is to determine the height of each node by the number of nodes it dominates. In graph-theoretic terminology, this corresponds to the indegree of the dominance relations. Figure2 2 illustrates the two drawings for the running example. 


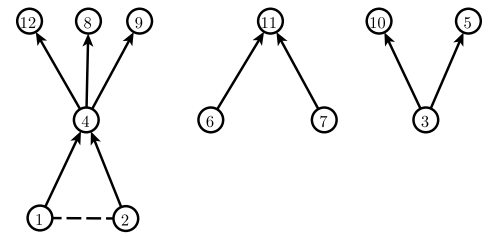

(a) Level-based drawing

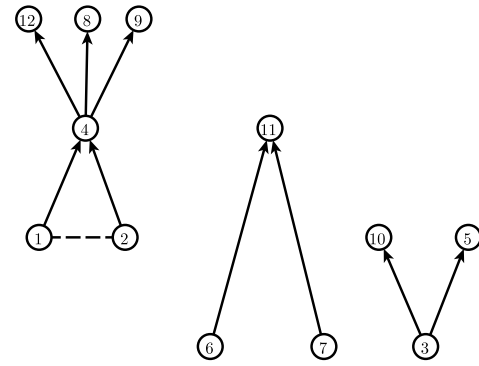

(b) Indegree-based drawing

Figure 2: Hasse diagram for the neighborhood-inclusion preorder of the running example. The dashed line indicates mutual dominance.

The set of maximal elements consists of 5, 8, 9, 10, 11 and 12. Any centrality index in accordance with Theorem 1 will rank one of these nodes on top. Recall from Table 1 that 12 has the highest degree, 10 the highest betweenness, 9 the highest closeness and 8 the highest eigenvector centrality. An index that would rank node 11 on top is subgraph centrality.

Hasse diagrams are a convenient representation if the number of nodes is comparatively small, but quickly become complicated and hard to interpret. Moreover, it does not explicitly capture potential rank ambiguities.

\subsection{Rank intervals}

To better evaluate rank ambiguities, we turn to rank intervals (Patil and Taillie, 2004). The rank interval of node $u \in V$ contains all potential ranks a node can occupy in a ranking that extends a given partial ranking. Its lower bound is given by $|\{w: w \leq u\}|+1$, that is $u$ is only ranked higher than the nodes it dominates. The upper bound is defined accordingly as $|V|-$ $|\{w: u \leq w\}|$. The size of each interval corresponds to the ambiguity inherent in attempting to rank specific nodes. The larger the interval, the higher the number of possible ranks. The mid-point of the interval should not be confused with an average or expected rank, since the ranks in each interval do not occur with uniform probability. The rank intervals based on neighborhood-inclusion for the running example are shown in Figure 3.

As a comparison, Figure 4 displays the rank intervals for dominance under total homogeneity for geodesic distances. Observe that the size of the intervals greatly decreased compared to neighborhood-inclusion. The intervals for nodes 1, 2, 3 and 5 even reduce to a single point. These nodes thus always occupy the same rank for indices that are based on monotonic transformations of geodesic distances. Additionally, only nodes 9 and 12 can be ranked on top. Thus, we will always expect a high rank correlation among distance based indices as already shown in Table 3 for a selection of such indices.

For each rank within an interval, there exists at least one ranking where the node occupies this rank. However, it is not straightforward to compute all such 


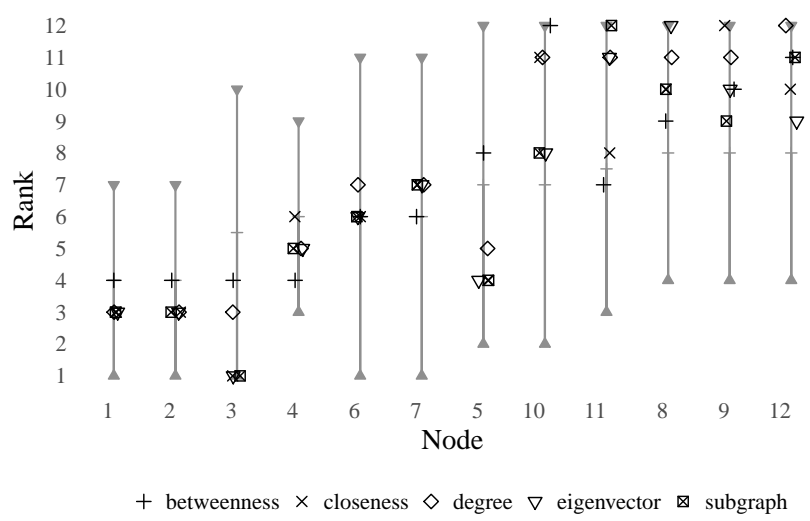

Figure 3: Rank intervals for the running example together with the ranks of each node for five indices. Nodes are arranged according to the midpoint of the intervals indicated with a horizontal line. We use the convention that high numerical ranks correspond to top ranked nodes.

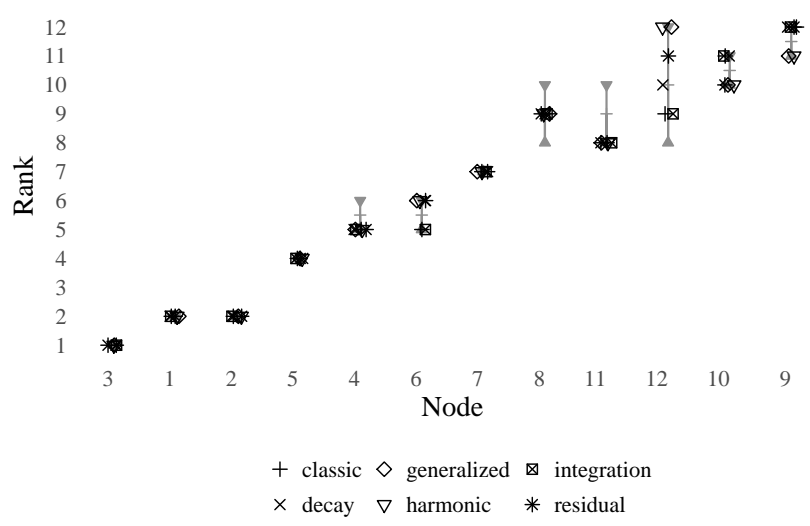

Figure 4: Rank intervals for the running example based on dominance under total homogeneity for geodesic distances together with the ranks of each node for indices from Table 22. Nodes are arranged according to the midpoint of the intervals indicated with a horizontal line. We use the convention that high numerical ranks correspond to top ranked nodes.

rankings and thus probabilities for each rank. We turn to this problem in the upcoming part.

\section{Probabilistic centrality rankings}

A partial ranking can be extended into a ranking by resolving all incomparable pairs of nodes. The set of potential rankings can be enormous depending on how many pairs are incomparable. For instance, if we dismiss the requirements of preserving the neighborhood-inclusion preorder, we would have around 500 
million $(\approx 12 !)$ potential centrality rankings for our running example. Centrality indices induce just one possible ranking, potentially making arbitrary decisions on how these incomparable nodes should be ordered. Even if the indirect relation is clear from the context, we can still define a range of indices since we only require the transformation function $\tau$ to be monotonic.

In this section, we present a more generic approach by considering all possible rankings that extend one of the given partial rankings by a probabilistic approach. We define the set of all rankings that preserve the neighborhoodinclusion preorder as

$$
\mathcal{R}(\leq)=\{r k: V \rightarrow\{1,2, \ldots, n\}: u \leq v \Longrightarrow r k(u) \leq r k(v)\},
$$

where $n=|V|$. We use the convention that the top ranked node $u$ is assigned $r k(u)=n$ and for the bottom ranked node $v$ we set $r k(v)=1$. For our running example, the set $\mathcal{R}(\leq)$ contains 221760 possible rankings. Analogously, $\mathcal{R}\left(\leq_{A^{*}}\right)$ is the set of rankings that extend dominance under total homogeneity on $A^{*}$. This set contains only 20 rankings for distance based indices on the example graph.

Known methods to determine all rankings and probabilities are rather involved and go beyond the scope of this paper. These methods and corresponding algorithms are, however, discussed in great detail in the supplementary fil $5^{5}$. Determining the set $\mathcal{R}(\leq)$ is a \#P complete problem, such that the computations quickly become infeasible Brightwell and Winkler (1991). The supplementary material thus also contains methods and algorithms for approximation.

\subsection{Rank probabilities}

Instead of insisting on a fixed rank, we can assess the probability for each node to occupy a specific rank $k$. This is simply done by counting all rankings where $r k(u)=k$ and dividing it with the total number of rankings. Formally, rank probabilities are defined as

$$
P(r k(u)=k)=\frac{|\{r k \in \mathcal{R}(\leq): r k(u)=k\}|}{|\mathcal{R}(\leq)|} .
$$

In terms of indices, these probabilities indicate how likely it is for a random centrality index to rank the node on a specific position. Most notable is the probability to be the most central node in a network, that is $P(r k(u)=n)$. Note that this probability is zero if there exists a node $v$ such that $u \leq v$. Table 4 shows the probabilities to be the top ranked node in the example graph for both considered partial rankings. Recall that we found five indices that each ranks a different node on top. The rank probabilities derived from the neighborhoodinclusion preorder now tell us how likely it is to find an index that rank specific nodes on top, given that we restrict the set of feasible indices to the ones that meet the requirements of Theorem 1 The overall picture changes significantly

\footnotetext{
${ }^{5}$ All methods are also available in the $\mathrm{R}$ package XXX blinded for review
} 
Table 4: Probability to be the most central node in the example network for neighborhoodinclusion and distance based dominance under total homogeneity. Only non-zero values are shown.

\begin{tabular}{crrrrrr}
\hline$P(r k(i)=n)$ & 5 & 8 & 9 & 10 & 11 & 12 \\
\hline$\leq$ & 0.14 & 0.15 & 0.15 & 0.14 & 0.27 & 0.15 \\
$\leq_{\text {dist }}$ & 0.00 & 0.00 & 0.80 & 0.00 & 0.00 & 0.20 \\
\hline
\end{tabular}

when considering the distance based dominance under total homogeneity. While the rank intervals already exposed node 9 and 12 as the only possible central nodes, the rank probabilities additionally reveal a strong tendency towards node 9.

\subsection{Expected Ranks}

The rank probabilities from above can be used to define the expected rank of nodes as

$$
\rho(r k(u))=\sum_{k=1}^{n} k \cdot P(r k(u)=k) .
$$

If all rankings are considered equally likely, a low expected rank points to a high likelihood that the node is among the least central nodes independent of the used index. A high expected rank on the other hand provides evidence that a node is always among the most central ones. In some sense, the expected ranks reflect a consensus ranking and the standard deviations a level of confidence. Additionally, it can be seen as a baseline ranking to compare index induced rankings to; the more an index deviates from the expectation, the more biased it is. A biased index, however, is not necessarily undesirable. It has to be checked carefully if the bias appears coincidentally or constitutes an acceptable result, which heavily depends on the research question.

The expected ranks together with the standard deviation of the running example for both considered partial rankings are shown in Figure 5 .

For neighborhood inclusion, we observe a high standard deviasions for each node, meaning that there is a low confidence in the expected ranks. The standard deviasions decrease strongly for the distance based dominance relation, such that the expected ranks reflect a reliable consensus ranking of the nodes based on distances. Note that although node 12 has non-zero probability to be the most central, its expected rank is lower than node 10's, which has zero probability to be the most central.

\subsection{Relative Rank Probabilities}

Instead of insisting on obtaining a ranking for all nodes, it can be sufficient to know the probability if a node $v$ is more central than a node $u$. These relative rank probabilities are defined as

$$
P(r k(u) \leq r k(v))=\frac{|\{r k \in \mathcal{R}(\leq): r k(u) \leq r k(v)\}|}{|\mathcal{R}(\leq)|}
$$



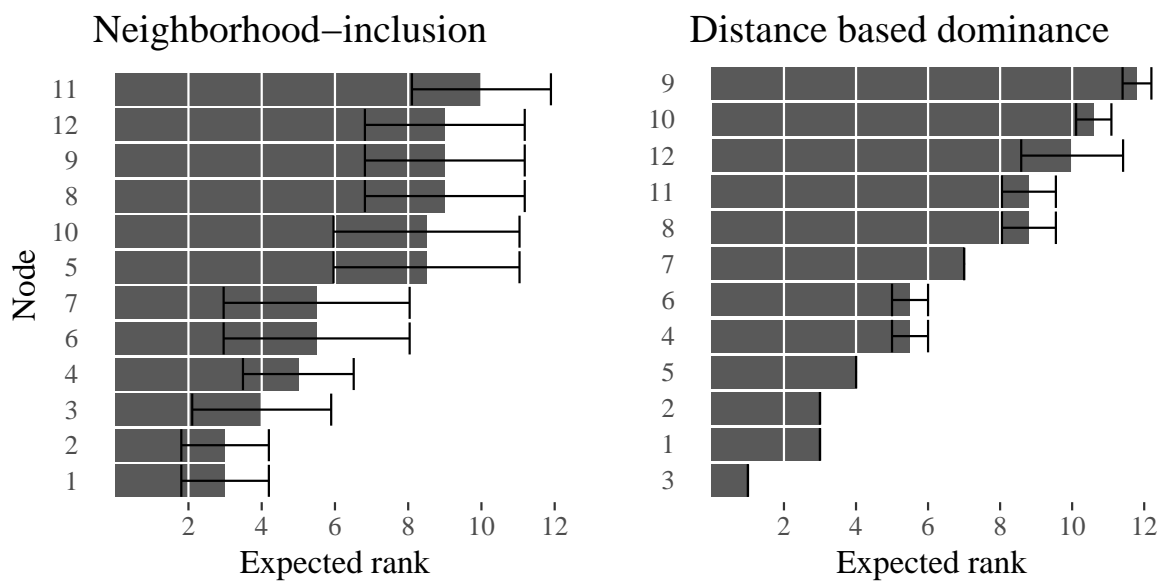

Figure 5: Expected ranks for the two considered partial rankings on the example graph. Error bars indicate the standard deviasion.

Note that $P(r k(u) \leq r k(v))=1$ if $u \leq v$. A probability higher than 0.5 suggests that it is preferable to rank $u$ below $v$. The more this probability deviates from 0.5 the stronger the degree of preference of $v$ over $u$. Table 5 shows the relative rank probabilities for the running example. Observe how

Table 5: Relative rank probabilities of the neighborhood-inclusion preorder for the running example.

\begin{tabular}{rrrrrrrrrrrrr}
\hline$\leq$ & 1 & 2 & 3 & 4 & 5 & 6 & 7 & 8 & 9 & 10 & 11 & 12 \\
\hline 1 & 0.00 & 0.00 & 0.62 & 1.00 & 0.94 & 0.76 & 0.76 & 1.00 & 1.00 & 0.94 & 0.98 & 1.00 \\
2 & 0.00 & 0.00 & 0.62 & 1.00 & 0.94 & 0.76 & 0.76 & 1.00 & 1.00 & 0.94 & 0.98 & 1.00 \\
3 & 0.38 & 0.38 & 0.00 & 0.64 & 1.00 & 0.65 & 0.65 & 0.91 & 0.91 & 1.00 & 0.95 & 0.91 \\
4 & 0.00 & 0.00 & 0.36 & 0.00 & 0.82 & 0.54 & 0.54 & 1.00 & 1.00 & 0.82 & 0.93 & 1.00 \\
5 & 0.06 & 0.06 & 0.00 & 0.18 & 0.00 & 0.24 & 0.24 & 0.54 & 0.54 & 0.50 & 0.65 & 0.54 \\
6 & 0.24 & 0.24 & 0.35 & 0.46 & 0.76 & 0.00 & 0.50 & 0.81 & 0.81 & 0.76 & 1.00 & 0.81 \\
7 & 0.24 & 0.24 & 0.35 & 0.46 & 0.76 & 0.50 & 0.00 & 0.81 & 0.81 & 0.76 & 1.00 & 0.81 \\
8 & 0.00 & 0.00 & 0.09 & 0.00 & 0.46 & 0.19 & 0.19 & 0.00 & 0.50 & 0.46 & 0.61 & 0.50 \\
9 & 0.00 & 0.00 & 0.09 & 0.00 & 0.46 & 0.19 & 0.19 & 0.50 & 0.00 & 0.46 & 0.61 & 0.50 \\
10 & 0.06 & 0.06 & 0.00 & 0.18 & 0.50 & 0.24 & 0.24 & 0.54 & 0.54 & 0.00 & 0.65 & 0.54 \\
11 & 0.02 & 0.02 & 0.05 & 0.07 & 0.35 & 0.00 & 0.00 & 0.39 & 0.39 & 0.35 & 0.00 & 0.39 \\
12 & 0.00 & 0.00 & 0.09 & 0.00 & 0.46 & 0.19 & 0.19 & 0.50 & 0.50 & 0.46 & 0.61 & 0.00 \\
\hline
\end{tabular}

close to 0.5 the probabilities for the maximal elements of the preorder are. It is thus hard to decide which node should be favored over which.

Table 6 shows the relative rank probabilities for the partial ranking of the distance based dominance under total homogeneity. The only remaining ambiguous pairs are node 4 and 6 , and node 8 and 11. All other pairs are mostly determined or the probabilities deviate strongly from 0.5 . 
Table 6: Relative rank probabilities of the distance based dominance under total homogeneity for the running example.

\begin{tabular}{rrrrrrrrrrrrr}
\hline$\leq_{\text {dist }}$ & 1 & 2 & 3 & 4 & 5 & 6 & 7 & 8 & 9 & 10 & 11 & 12 \\
\hline 1 & 0.00 & 0.00 & 0.00 & 1.00 & 1.00 & 1.00 & 1.00 & 1.00 & 1.00 & 1.00 & 1.00 & 1.00 \\
2 & 0.00 & 0.00 & 0.00 & 1.00 & 1.00 & 1.00 & 1.00 & 1.00 & 1.00 & 1.00 & 1.00 & 1.00 \\
3 & 1.00 & 1.00 & 0.00 & 1.00 & 1.00 & 1.00 & 1.00 & 1.00 & 1.00 & 1.00 & 1.00 & 1.00 \\
4 & 0.00 & 0.00 & 0.00 & 0.00 & 0.00 & 0.50 & 1.00 & 1.00 & 1.00 & 1.00 & 1.00 & 1.00 \\
5 & 0.00 & 0.00 & 0.00 & 1.00 & 0.00 & 1.00 & 1.00 & 1.00 & 1.00 & 1.00 & 1.00 & 1.00 \\
6 & 0.00 & 0.00 & 0.00 & 0.50 & 0.00 & 0.00 & 1.00 & 1.00 & 1.00 & 1.00 & 1.00 & 1.00 \\
7 & 0.00 & 0.00 & 0.00 & 0.00 & 0.00 & 0.00 & 0.00 & 1.00 & 1.00 & 1.00 & 1.00 & 1.00 \\
8 & 0.00 & 0.00 & 0.00 & 0.00 & 0.00 & 0.00 & 0.00 & 0.00 & 1.00 & 1.00 & 0.50 & 0.70 \\
9 & 0.00 & 0.00 & 0.00 & 0.00 & 0.00 & 0.00 & 0.00 & 0.00 & 0.00 & 0.00 & 0.00 & 0.20 \\
10 & 0.00 & 0.00 & 0.00 & 0.00 & 0.00 & 0.00 & 0.00 & 0.00 & 1.00 & 0.00 & 0.00 & 0.40 \\
11 & 0.00 & 0.00 & 0.00 & 0.00 & 0.00 & 0.00 & 0.00 & 0.50 & 1.00 & 1.00 & 0.00 & 0.70 \\
12 & 0.00 & 0.00 & 0.00 & 0.00 & 0.00 & 0.00 & 0.00 & 0.30 & 0.80 & 0.60 & 0.30 & 0.00 \\
\hline & & & & & & & & & & & &
\end{tabular}

\section{Application}

In this section, we illustrate how the presented methododology can be used in empirical context. We consider the network of marriages among Florentine families during the Renaissance, one of the classic datasets in social network analysis, shown in Figure 6(a). Its structure reveals how the Medici family could rise to power without being the richest or having the most seats in the local legislature (Padgett and Ansell, 1993). The network is often used as a benchmark for centrality indices (Wasserman and Faust, 1994, Bonacich and Lloyd, 2001, Newman, 2005, Estrada et al., 2009), since the Medici are supposed to occupy a central position due to skillful manipulation of marriages. Reasonable centrality indices are thus expected to rank the Medici on top or at least higher than their arch-rivals, the Strozzi.

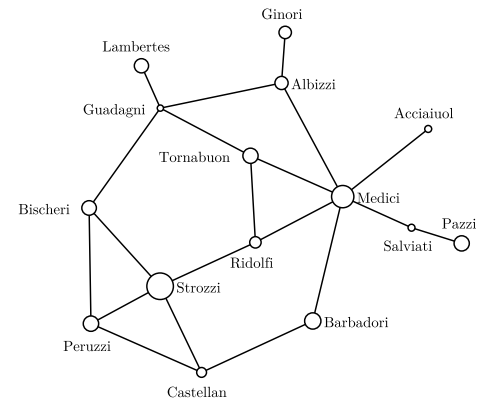

(a) Marriage ties.

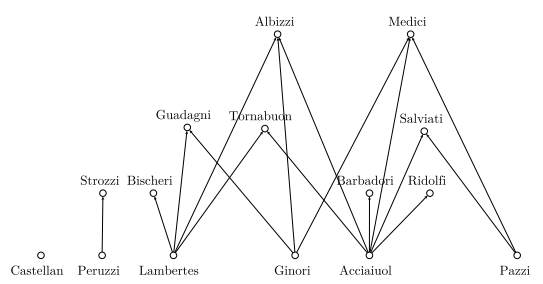

(b) Neighborhood-inclusion preorder.

Figure 6: Marriage network of 15 Florentine families. Size of node proportional to the wealth attribute. (The Pucci family is removed since they are isolated.) 


\subsection{Neighborhood-inclusion}

The partial ranking induced by neighborhood-inclusion can be used to obtain a broad overview about centrality in the network and assess to which extend it is meaningful to work with indices.

In the network, $85 \%$ of pairs of families are incomparable and 10 of the families are not dominated by any other, such that there exist rankings that rank any of these on top. The Strozzi and Medici are incomparable, meaning that there exist rankings that prefer one or the other.

The rank intervals, shown in Figure 7, give a first hint to the freedom for obtaining rankings. Notice that all prototypical indices rank the Medici on top,

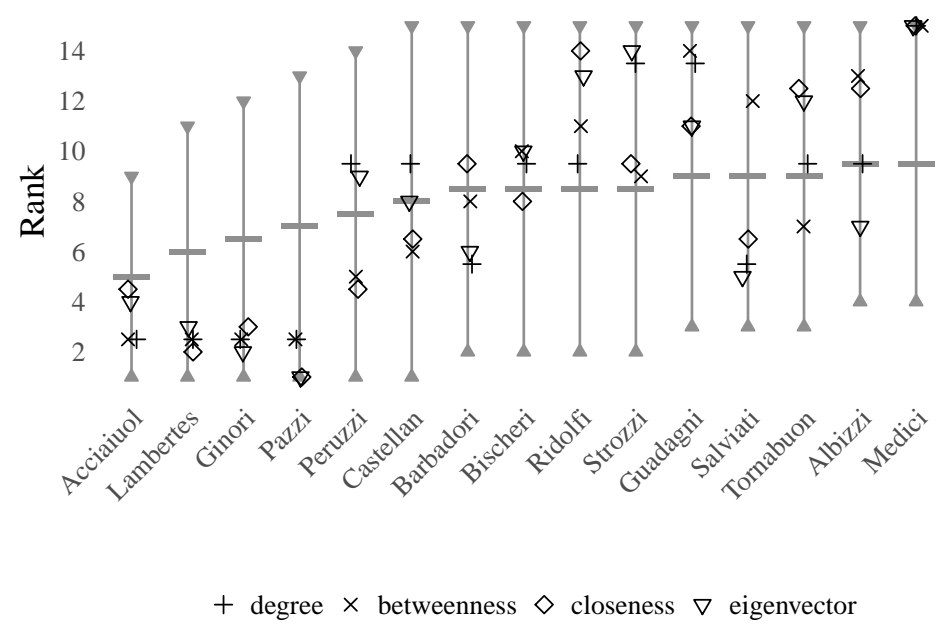

Figure 7: Rank intervals together with the rank of the prototypical centrality indices for Florentine families based on neighborhood-inclusion.

yet it is well possible to find indices that rank them lower. An example is odd subgraph centrality (Estrada and Rodríguez-Velázquez, 2005a), defined as

$$
c_{s o}(u)=\sum_{k=0}^{\infty} \frac{A(G)_{u u}^{2 k+1}}{(2 k+1) !},
$$

which ranks the Strozzi on top and the Medici on third position.

The set $\mathcal{R}(\leq)$ of all centrality rankings contains almost 4 billion possibilities, amplifying the huge freedom for indices to rank the families differently. Among those, the Medici are considered to be the most central in $12.3 \%$ of all cases. The Strozzi family, with $13.3 \%$, is slightly more likely to be on top. Yet, if we consider relative rank probabilities, the probability that the Medici are more central than the Strozzi is 0.52 , such that the Medici are slightly favored over the Strozzi. Figure 8 shows the complete rank probability distributions of families together with the expected ranks and standard deviation. As anticipated, the 


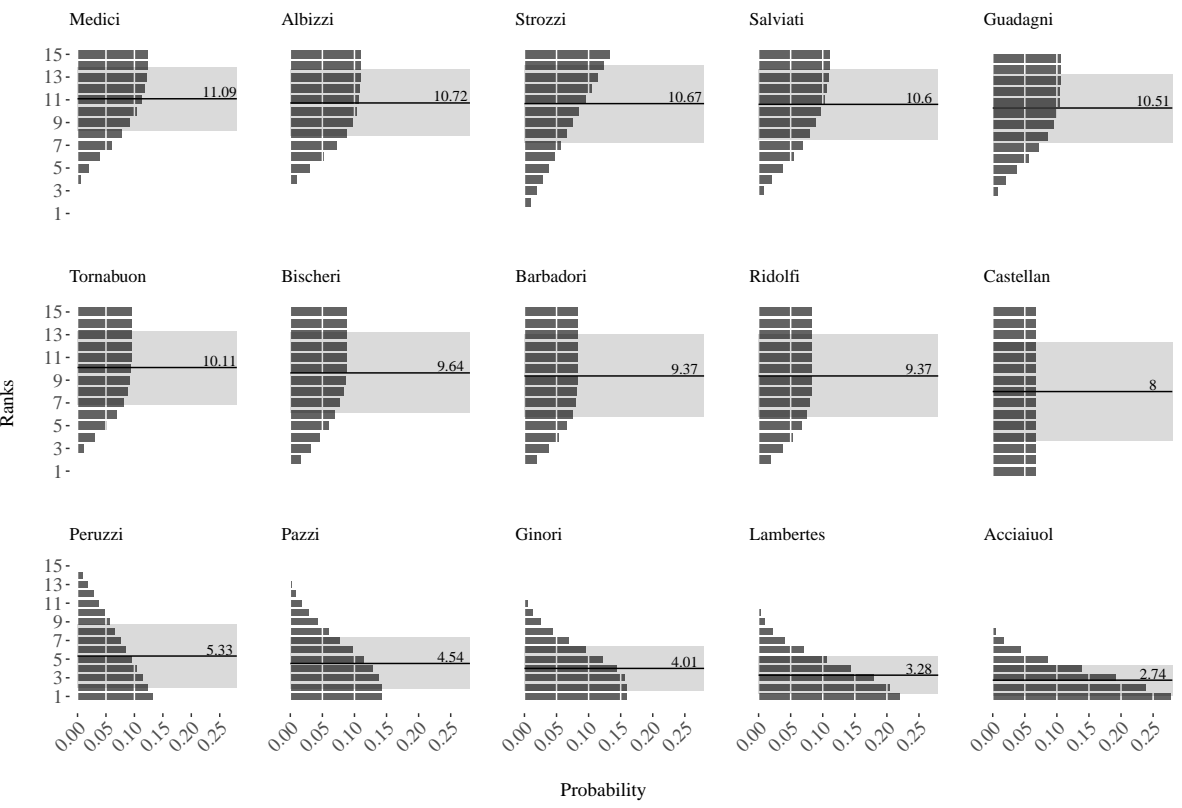

Figure 8: Rank probability distribution of families sorted by the expected rank (black line). The grey area indicates the standard deviasion of the expected rank.

Medici are expected to be the most central family in the network, if only by a small margin. The uniform rank probabilities of the Castellan family stem from the fact that they are neither dominated nor dominate any other family (cf.Figure 6(b)), such that every rank is equally likely.

The results show that the scope for centrality rankings is enormous. While the most prominent indices indeed rank the Medici on top, there exists enough degree of freedom to allow for other results, as illustrated by odd subgraph centrality.

\subsection{Dominance under total homogeneity}

In order to reasonably work with dominance under total homogeneity, we first need more concrete ideas about processes that make nodes in certain positions more central. A specific hypothesis for the Florentine families could be that (geodesic) distances in the marriage network serve as an indicator for the wealth of families. Closer relations to all families offer more opportunities to do business and thus to increase wealth. Additionally, we assume that relations to specific others does not matter and only overall small distance are of importance. Hence, we can make use of the homogeneity assumption and reorder the distance based positional vectors non-increasingly. This allows us to extend the neighborhood-inclusion preorder to dominance under total homogeneity for geodesic distances. Before we employ the presented methods, we investigate the hypothesis using an index-driven approach to illustrate potential problems. 
We start by employing classic closeness to check for an association between wealth and its scores. We obtain a weak rank correlation 6 of 0.09 and an association between "being close" to all families and wealth can not be established. As we have seen in Section 2.4, there exist additional distance based indices that could be used to find a better association. The rank correlation among indices from Table 2 and the wealth attribute in Table 7 shows, however, that all indices are only weakly associated with the wealth attribute.

Table 7: Rank Correlation among distance based indices and the wealth attribute. Values in parenthesis indicate parameter settings that produce the maximal correlation with wealth.

\begin{tabular}{llllllll}
\hline & $(1)$ & $(2)$ & $(3)$ & $(4)$ & $(5)$ & $(6)$ & $(7)$ \\
\hline (1) closeness & 1.00 & & & & & & \\
(2) harmonic & 0.86 & 1.00 & & & & & \\
(3) integration & 1.00 & 0.86 & 1.00 & & & & \\
(4) residual & 0.91 & 0.95 & 0.91 & 1.00 & & & \\
(5) decay $(\alpha=0.26)$ & 0.79 & 0.95 & 0.79 & 0.89 & 1.00 & & \\
(6) generalized $(\alpha=0.51)$ & 0.91 & 0.95 & 0.91 & 1.00 & 0.89 & 1.00 & \\
\hline (7) wealth & 0.09 & 0.10 & 0.09 & 0.12 & 0.12 & 0.12 & 1.00 \\
\hline
\end{tabular}

We could now either accept this result or proceed to find a distance based index that potentially gives a higher correlation. This amounts to finding a monotonic transformation function $\tau$ such that its summarisation induces a ranking close to the ranking induced by wealth. Possibilities are almost endless and we may run the risk of ending up in one of the following scenarios. If we find a transformation that produces a good fit, there may still be a transformation with an even better fit. On the other hand, if we do not find a good fit, we can not guarantee its non-existence.

Analyzing the partial ranking induced by distance based dominance total homogeneity allows us to examine all possible rankings and check if a potential better fit could exist. The number of incomparable pairs of families under these circumstances decreases to only $18 \%$. Thus, there is actually little freedom for distance based indices to rank nodes differently. The Hasse diagram for the dominance relations is shown in Figure 9. It becomes evident that no distance based index is able to perfectly explain wealth. Many wealthy families are dominated by poorer ones, indicated by the gray edges in the figure. The Guadagni family with the lowest value in wealth, for instance, dominates the Strozzi, the wealthiest family in the dataset. Moreover, the only undominated family are the Medici such that they occupy the top position in any possible distance based ranking. This can also be seen in Figure 10, where the rank interval decreases to a single point for the Medici. Comparing these rank intervals with Figure 7, we see how the decision of using geodesic distances and the homogeneity assump-

${ }^{6}$ We use Kendall's $\tau$ to measure rank correlation 


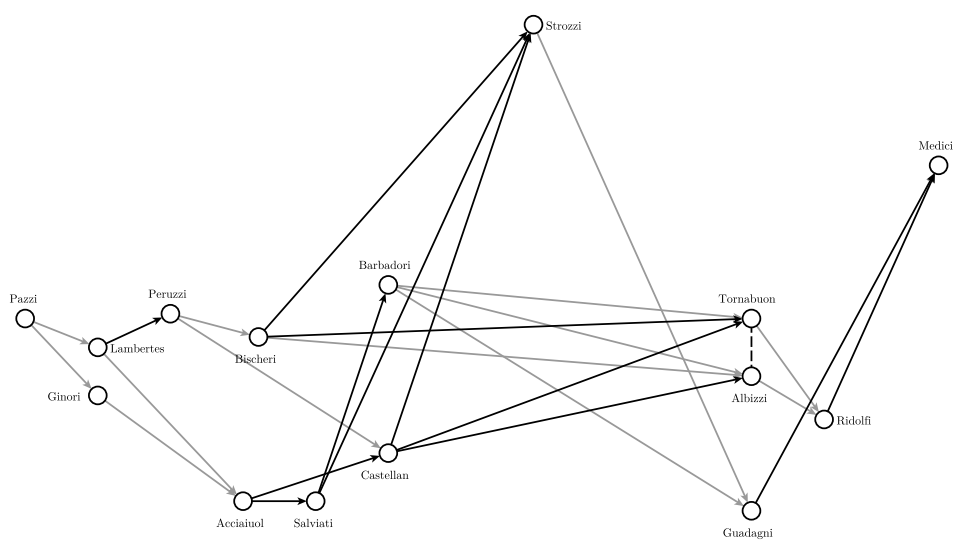

Figure 9: Alternative Hasse diagram of dominance under total homogeneity for the distance relation among Florentine families. The $x$-coordinate of nodes is proportional to the number of dominating families and the $y$-coordinate to the wealth attribute. Edges in gray indicate relations where a wealthy family is dominated by a less wealthy one.

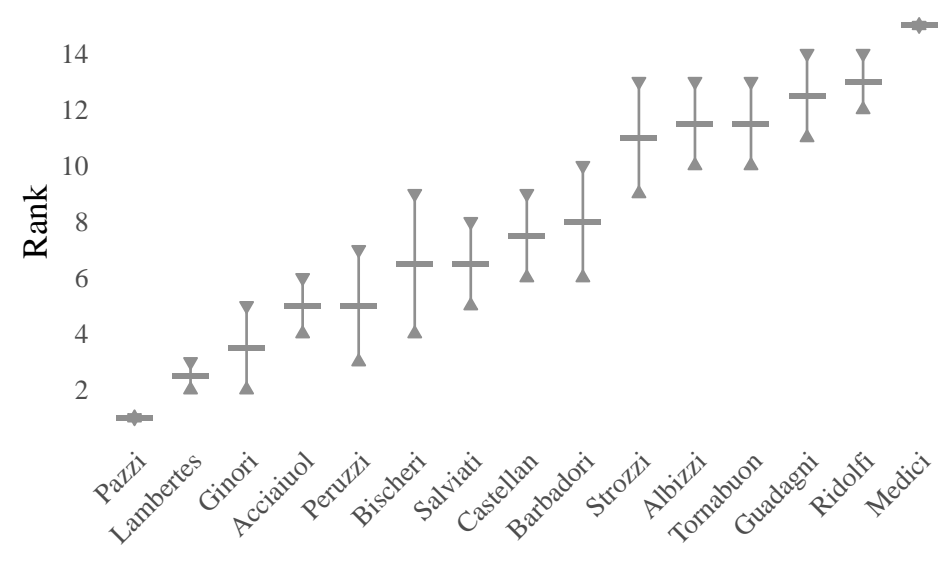

Figure 10: Rank intervals of Florentine families for dominance under total homogeneity of geodesic distances.

tion reduced the set of possible ranks for each family. This becomes even more clear when looking at the set of all rankings $\mathcal{R}\left(\leq_{\text {dist }}\right)$, which contains only 654 rankings. This constitutes an enormous contraction of $\mathcal{R}(\leq)$. The maximal possible rank correlation between any of these distance rankings and wealth is 0.15 . This indicates that no centrality index derived from a monotonic transformation of geodesic distances can obtain a higher correlation with the wealth attribute. On top of that, this upper bound is lower than the rank correlation of wealth with degree (0.20). We can thus conclude, that there does not exist a distance 
based index that can reasonably explain the wealth attribute. Moreover, the higher correlation of degree with wealth indicates that direct marriage ties are more important than being close (in graph-theoretic terms) to other families. This conclusion was already reached by Agneessens et al. (2017), however, the presented results are more rigorous since it is guaranteed that no such distance based index exists.

\section{Discussion}

The main purpose of this work was to introduce existing methods to analyze partial orders for network centrality. We illustrated that these tools can complement but also replace traditional index based assessment of centrality. While several arguments against the application of indices were made, it must be pointed out that their usage is certainly not universally problematic. If the application of a specific index is properly justified, there is nothing wrong with using it. Yet singling out indices by trial and error, choosing the one closest to the desired result should be avoided.

The partial centrality approach offers a way to gradually incorporate assumptions to systematically reduce the set of incomparable pairs, as it was illustrated with the Florentine family dataset. Nodes that are incomparable by neighborhood-inclusion might become comparable when further properly motivated assumptions are made. This encourages to move away from choosing or crafting indices toward carefully investigating which processes render nodes more central than others in specific situations. So far, the choice of relation is implicitly done by choosing indices, like geodesic distances for closeness. The identification of the relevant relation or (social) process to a particular context is, however, a non-trivial task and should be seen as part of any analysis and problem formulation process. This is enhanced by the observation that many processes of empirical interest are still not covered by any index while others are overrepresented (Borgatti, 2005).

Much effort has been put into crafting increasingly complicated indices, such that choosing appropriate measures has become an arduous task. Breaking down indices into a series of microsteps allows us to gradually incorporate substantive ideas as compared to using prefabricated bundles as offered by indices.

A non-negligible limitation of this work is that the preservation of neighborhoodinclusion is not an established foundation for network centrality as a whole. So far, only certain groups of existing indices are known to fulfill the strict requirements of Theorem 11. Thus, the scope of applicability is still limited and further research is needed to extend this scope. Yet, the presented results show that if the existence of intrinsic node orderings is acknowledged, it can prove helpful to analyse centrality with a different set of tools.

\section{Bibliography}

Agneessens, F., Borgatti, S. P., Everett, M. G., 2017. Geodesic based centrality: Unifying the local and the global. Social Networks 49, 12-26. 
Anthonisse, J. M., 1971. The rush in a directed graph. Tech. Rep. BN 9/71, Mathematical Centre, Amsterdam.

Bartel, H.-G., Mucha, H.-J., 2014. Measures of incomparability and of inequality and their applications. In: Multi-indicator Systems and Modelling in Partial Order. Springer-Verlag, pp. 47-67.

Batagelj, V., 1994. Semirings for social network analysis. Journal of Mathematical Sociology 19 (1), 53-68.

Bavelas, A., 1948. A mathematical model for group structures. Human Organizations 7 (3), 16-30.

Belton, V., Gear, T., 1983. On a short-coming of saaty's method of analytic hierarchies 11 (3), 228-230.

Belton, V., Stewart, T. J., 2002. Multiple criteria decision analysis: an integrated approach. Springer Science \& Business Media.

Benzi, M., Klymko, C., 2013. Total communicability as a centrality measure. Journal of Complex Networks 1 (2), 124-149.

Berge, C., 1958. Théorie des graphes et ses applications. Paris, France.

Boldi, P., Vigna, S., 2014. Axioms for centrality. Internet Mathematics 10 (3-4), 222-262.

Bonacich, P., 1972. Factoring and weighting approaches to status scores and clique identification. Journal of Mathematical Sociology 2, 113-120.

Bonacich, P., Lloyd, P., 2001. Eigenvector-like measures of centrality for asymmetric relations. Social Networks 23 (3), 191-201.

Borgatti, S. P., 2005. Centrality and network flow. Social Networks 27 (1), 5571.

Borgatti, S. P., Everett, M. G., 2006. A graph-theoretic perspective on centrality. Social Networks 28 (4), 466-484.

Brandes, U., 2016. Network positions. Methodological Innovations 9, 1-19.

Brightwell, G., Winkler, P., 1991. Counting linear extensions. Order 8 (3), 225242.

Brüggemann, R., Carlsen, L., 2014. Incomparable-what now? MATCH Commun. Math. Comput. Chem. 71, 694-716.

Brüggemann, R., Lerche, D., Sørensen, P. B., 2003. First attempts to relate structures of hasse diagrams with mutual probabilities. Technical Report 479, The 5th workshop held at the National Environmental Research Institute (NERI), 7-52. 
Brüggemann, R., Simon, U., Mey, S., 2005. Estimation of averaged ranks by extended local partial order models. MATCH Commun. Math. Comput. Chem. $54,489-518$.

Brüggemann, R., Sørensen, P. B., Lerche, D., Carlsen, L., 2004. Estimation of averaged ranks by a local partial order model. Journal of Chemical Information and Computer Sciences 44 (2), 618-625.

Bubley, R., Dyer, M., 1999. Faster random generation of linear extensions. Discrete Mathematics 201 (1), 81-88.

Dangalchev, C., 2006. Residual closeness in networks. Physica A: Statistical Mechanics and its Applications 365 (2), 556-564.

Davey, B. A., Priestley, H. A., 2002. Introduction to lattices and order. Cambridge University Press.

De Loof, K., 2009. Efficient computation of rank probabilities in posets. Ph.D. thesis, Ghent University.

De Loof, K., De Baets, B., De Meyer, H., 2008. Properties of mutual rank probabilities in partially ordered sets. In: Multicriteria Ordering and Ranking: Partial Orders, Ambiguities and Applied Issues. Systems Research Institute, Polish Academy of Sciences, pp. 145-165.

De Loof, K., De Meyer, H., De Baets, B., 2006. Exploiting the lattice of ideals representation of a poset. Fundamenta Informaticae $71(2,3), 309-321$.

Estrada, E., Higham, D. J., Hatano, N., 2009. Communicability betweenness in complex networks. Physica A: Statistical Mechanics and its Applications 388 (5), 764-774.

Estrada, E., Rodríguez-Velázquez, J. A., 2005a. Spectral measures of bipartivity in complex networks. Physical Review E 72 (4), 046105.

Estrada, E., Rodríguez-Velázquez, J. A., 2005b. Subgraph centrality in complex networks. Physical Review E 71 (5), 056103.

Fishburn, P. C., Gehrlein, W. V., 1975. A comparative analysis of methods for constructing weak orders from partial orders. Journal of Mathematical Sociology 4 (1), 93-102.

Freeman, L. C., 1977. A set of measures of centrality based on betweenness. Sociometry 40 (1), 35-41.

Freeman, L. C., 1979. Centrality in social networks: Conceptual clarification. Social Networks 1 (3), 215-239.

Gondran, M., Minoux, M., 2008. Graphs, Diods and Semirings. Springer-Verlag.

Grätzer, G., 2002. General lattice theory. Springer Science \& Business Media. 
Habib, M., Medina, R., Nourine, L., Steiner, G., 2001. Efficient algorithms on distributive lattices. Discrete Applied Mathematics 110 (2), 169-187.

Hage, P., Harary, F., 1995. Eccentricity and centrality in networks. Social Networks 17, 57-63.

Harris, C. D., 1954. The market as a factor in the localization of industry in the united states. Annals of the Association of American Geographers (4), $315-348$.

Jackson, M. O., 2010. Social and economic networks. Princeton University Press.

Janicki, R., 2008. Ranking with partial orders and pairwise comparisons. In: International Conference on Rough Sets and Knowledge Technology. Springer, pp. $442-451$.

Janicki, R., 2009. Pairwise comparisons based non-numerical ranking. Fundamenta Informaticae 94 (2), 197-217.

Katz, L., 1953. A new status index derived from sociometric analysis. Psychometrika $18(1), 39-43$.

Kitti, M., 2012. Axioms for centrality scoring with principal eigenvectors. Tech. Rep. 79, Aboa Centre for Economics, Turku University.

Landherr, A., Friedl, B., Heidemann, J., 2010. A critical review of centrality measures in social networks. Business \& Information Systems Engineering $2(6), 371-385$.

Lü, L., Chen, D., Ren, X.-L., Zhang, Q.-M., Zhang, Y.-C., Zhou, T., 2016. Vital nodes identification in complex networks. Physics Reports 650, 1-6.

Neggers, J., Kim, H. S., 1998. Basic Posets. World Scientific.

Newman, M. E. J., 2005. A measure of betweenness centrality based on random walks. Social Networks 27 (1), 39-54.

Nieminen, J., 1974. On the centrality in a graph. Scandinavian Journal of Psychology $15,332-336$.

Padgett, J. F., Ansell, C. K., 1993. Robust action and the rise of the medici 1400-1434. American Journal of Sociology 98 (6), 1259-1319.

Patil, G. P., Taillie, C., 2004. Multiple indicators, partially ordered sets, and linear extensions: Multi-criterion ranking and prioritization. Environmental and Ecological Statistics 11 (2), 199-228.

Pavan, M., Todeschini, R., 2004. New indices for analysing partial ranking diagrams. Analytica Chimica Acta 515 (1), 167-181.

Ruhnau, B., 2000. Eigenvector-centrality-a node-centrality? Social Networks $22(4), 357-365$. 
Sabidussi, G., 1966. The centrality index of a graph. Psychometrika 31 (4), 581-603.

Schoch, D., Brandes, U., 2016. Re-conceptualizing centrality in social networks. European Journal of Applied Mathematics 27 (6), 971-985.

Schoch, D., Valente, T. W., Brandes, U., 2017. Correlations among centrality indices and a class of uniquely ranked graphs. Social Networks 50, 46-54.

Stewart, T. J., 1992. A critical survey on the status of multiple criteria decision making theory and practice. Omega 20 (5-6), 569-586.

Triantaphyllou, E., 2013. Multi-criteria decision making methods: a comparative study. Vol. 44. Springer Science \& Business Media.

Triantaphyllou, E., Mann, S. H., 1989. An examination of the effectiveness of multi-dimensional decision-making methods: a decision-making paradox. Decision Support Systems 5 (3), 303-312.

Valente, T. W., Foreman, R. K., 1998. Integration and radiality: Measuring the extent of an individual's connectedness and reachability in a network. Social Networks 20 (1), 89-105.

Wasserman, S., Faust, K., 1994. Social Network Analysis. Methods and Applications. Cambridge University Press, Cambridge, UK.

Wei, T.-H., 1952. Algebraic foundations of ranking theory. Ph.D. thesis, University of Cambridge. 